\title{
Conception et déroulement du sacrifice chez les Mofu (Cameroun du Nord)
}

The conception and course of sacrifices among the Mofu (northern Cameroon)

Jeanne-Françoise Vincent

\section{OpenEdition}

Édition électronique

URL : http://journals.openedition.org/span/340

DOI : $10.4000 /$ span.340

ISSN : 2268-1558

Éditeur

École pratique des hautes études. Sciences humaines

\section{Édition imprimée}

Date de publication : 1 septembre 1976

Pagination : 177-203

ISSN : 0294-7080

\section{Référence électronique}

Jeanne-Françoise Vincent, « Conception et déroulement du sacrifice chez les Mofu (Cameroun du Nord) », Systèmes de pensée en Afrique noire [En ligne], 2 | 1976, mis en ligne le 04 juin 2013, consulté le 19 avril 2019. URL : http://journals.openedition.org/span/340 ; DOI : 10.4000/span.340 


\author{
CON C E P T I O N E T D ÉRO O L E M E N T \\ DU SACRIFICE C HEZ LES M O F U \\ Carneroun du Nord \\ par \\ Jeanne--Françoise Vincent
}

Chez les Mofu, le sacrifice apparaît comme un acte extrêmement courant, pour ne pas dire banal. Au début de mes missions en pays mofu il $\mathrm{m}^{\prime}$ est arrivé en saison sèche de passer des jours entiers à aller de sacrifice en sacrifice. Ma documentation sur ce thème précis est donc abondante, comportant à la fois des descriptions théoriques, partielles le plus souvent, et des observations directes portant sur une soixantaine de sacrifices.

Cependant, une description valable du "sacrifice mofu" est impossible : la diversité du panthêon mofu entrâ̂ne en effet une pluralité de schëmas sacrificiels correspondant, semble-t-il, à divers niveaux d'anciennetê. Le cadre limité de cette communication interdisant de passer en revue ces pratiques en montrant leurs convergences et leurs divergences, je me contenterai pour commencer de dêfinir le sacrifice mofu, en essayant de trouver un tronc commun à tous les sacrifices et de rendre compte des esprits auxquels ils s'adressent. Ensuite, en illustration, je décrirai un sacrifice mofu, le sacrifice aux ancêtres, en faisant référence, chaque Ẽois que cela sera utile, à 1'essai de H. Hubert et M. Mauss sur le sacrifice (1) qui m'a souvent servi de guide de réflexion.

(1) H. HUBERT et M. MAUSS, Essai sur la nature et la fonction du sacrifice, L'année sociologique, I (1899), pp. 29-138. 
I. Préambules à une étude du sacrifice mofu.

A. Essai de définition.

En l'absence d'une définition élaborée par Les Mofu eux-mêmes, il est possible de partir de l'étude de leurs mots pour essayer de cerner la réalité du sacrifice (2). On découvre d'abord une abondance de termes particuliers, descriptifs et concrets, s'appliquant chacun à un type de sacrifice ou à une étape d'un cycle de sacrifices et signifiant par exemple "commencer", "mettre en place", "laver les mains", "changer de bouche", etc. Toutefois, lorsqu'il s'agit d'un sacrifice ordinaire, sans spécification, on emploie un terme plus large, correspondant à "offrir", "poser des offrandes" : meteuley. Il existe également une notion qui correspond parfois à celle de sacrifice, pris dans un sens général, c'est celle de kuZey. Mais il s'agit là d'un terme élastique dont le champ sêmantique est large, recouvrant divers autres sens, celui de sacrifice n'êtant pas le plus immédiat. Suivant le contexte, kuley ( $k u l i$ au pluriel) peut désigner en effet un esprit d'ancêtre, et c'est là son sens le plus courant : "Après la mort, les kuli continuent à se promener au milieu des vivants, seulement on ne les voit pas." (3). "Avec le mil que j'ai récoltê, je ne peux pas faire actuellement de 1 a bière pour vendre, sinon les $k u l_{i}$ se fâcheraient contre moi et diraient que je gaspille le mil." (4). kuley désigne aussi un autel consacré à un ancêtre. Ohunom, de Durum, m'invitant à voir ses autels familiaux, les désignait un à un : "Voici le kuley de mon père; 1à, c'est le kuley de sa mère." (5). kuley peut aussi correspondre à "charme magique". On le rencontre avec ce sens lors des consultations divinatoires : le devin, examinant les causes de perturbation possibles, réserve une pierre à ce qu'il appelle kuli ma ndo,

(2) Cette étude se limitera aux massifs de Duvangar, Durum et Wazan, représentant 13000 personnes parlant la même langue.

(3) Information de Pemley (70 ans ?), Wazan, 6/1/1969.

(4) Dizeleng (ferme de 38 ans ?), Wazan, 15/12/1969.

(5) Ohunom (75 ans ?), Durum, 10/1/1968. 
"charme magique de quelqu'un" (déposé par quelqu'un) (6). Enfin kuley désigne le sacrifice : meki kuley signifie "faire un sacrifice" ; le ndo kuley est "l'homme de sacrifice", celui qui aide chaque "chef de maison" à célébrer son culte familial ; kuli doit être traduit par "sacrifice" dans le proverbe signifiant "il ne faut jamais remettre au lendemain" :

kuli ma aza
sacrifice de plus tard ils font est-ce-que?

kuli désigne donc tout ce qui a trait au surnaturel et peut ainsi s'appliquer à l'acte par lequel les hommes entrent en contact avec le domaine du surnaturel.

L'examen des termes amène à penser que, pour les Mofu, le sacrifice est une offrande qui introduit dans le domaine du surnaturel. L'observation des sacrifices -- des plus simples aux plus complexes -invite à préciser cette notion en la complétant par celle de destruction et celle de paroles. Le sacrifice mofu consiste en une offrande effectuée en faveur d'un esprit supranaturel, comportant une destruction partielle et accompagnée de paroles adressées à cet esprit par un ou plusieurs célêbrants. Il y a union indissociable entre 1'action religieuse et la prière, "les deux moitiés du rite complet", suivant 1'expression d'É. Benveniste, qui ajoute : "les deux moyens d'accéder au monde divin" (7). Le sacrifice mofu apparaît bien comme un mode de mise en communication de deux domaines distincts, de 'Geux 'récipients' qui ne sont pas au même niveau", ainsi que le souligne cl. Lévi-Strauss (8) pour qui le sacrifice est une sorte d'écluse.

B. Les esprits destinataires.

H. Hubert et M. Mauss 'considèrent que 1'essentiel du sacrifice réside dans la modification du sacrificateur et de l'offrande. Ils se

(6) J.-F. VINCENT, Divination et possession chez les Mofu, montagnards du Nord-Cameroun, $J$. Soc. Africanistes, XLI, 1 (1971), pp. 71132 ; cf. pp. 90-91.

(7) É. BENVENISTE, Le vocabulaire des institutions indo-européennes (Paris, Éd. de Minuit, 1969) ; cf. II, p. 245.

(8) C1. LÉvi-STRAuSS, La persée sauvage (Paris, Plon, 1962); cf. p. 298 . 
limitent délibérêment aux seuls aspects visibles du sacrifice, négligeant les esprits destinataires qui apparaissent comme les grands absents de leur étude. Il nous paraît cependant nécessaire, avant de classer et, à plus forte raison, de décrire les sacrifices, de dresser un inventaire des esprits auxquels s'adresse 1a société ; chez les Mofu, la démarche s'impose car l'identité de l'esprit destinataire de 1 'offrande conditionne la forme extérieure de celle-ci.

Les ancêtres peuvent être considêrés -- du seul point de vue statistique -- comme les esprits supra-naturels jouant le rôle le plus important dans la vie religieuse des Mofu. La vivacité de la croyance en leur pouvoir se traduit par un culte très développé et diversifié qui éclipse presque les autres cultes.

Tout homme adulte -- ou "chef de maison", $b i$ ay -- entretient en effet un culte à sept ancêtres distincts ayant avec lui une relation généalogique directe : son père dêfunt, ses grand-père et arrière grand-père, tous trois ayant leur autel au même endroit, au coeur de 1'habitation, dans la salle des greniers; le père de l'arrière grandpère, qui constitue un véritable esprit-garđien, possède, 1ui, un autel placê près du seuil de l'habitation ; à ces quatre ancêtres masculins de la maison, il faut ajouter deux ancêtres féminins, la mère défunte du chef de famille et la mère de son père. Un septième ancêtre -- cinquième ancêtre masculin -- est encore révéré ; il s'agit de l'esprit des champs de mil, père de l'esprit de la porte, dont l'autel est situé cette fois à l'extérieur de la maison, au milieu de la plantation principale de mil. On voit donc la profondeur généalogique particulière de ce culte des ancêtres. En plus de ces esprits distincts, dont chacun peut recevoir un sacrifice pour lui seul, un chef de famille peut sacrifier aux frères de son père, morts jeunes et sans descendance; ces derniers reçoivent par ailleurs une part collective lors des sacrifices importants offerts à un ancêtre direct.

$\mathrm{Au}$ fur et à mesure que ces ancêtres individuels s'éloignent des vivants, ils ne sont pas totalement oubliés; on les honore collectivement en un autel détenu par I'aîné đu lignage.

Les esprits susceptibles de nuire donnent lieu à trois types de cultes individuels, beaucoup moins répandus que le culte des ancêtres et non-transmissibles, dont la caractéristique commune est qu'ils sont rendus sous la contrainte afin d'obtenir la neutralisation des esprits. 
Les cultes aux esprits d'hommes tués sont en voie de disparition mais ils étaient très répandus autrefois où les guerres étaient fréquentes. Celui qui a été tué -- pendant une guerre ou à la suite d'une rixe -- peut nuire à son meurtrier et il est nécessaire de $1^{\text {' }}$ apaiser par des sacrifices. Si plusieurs personnes ont contribué à cette mort, le culte est collectif et cesse avec la disparition du dernier participant. Les esprits d'animaux tués -- biches-cochon, antilopes, antilopes-cheval, panthères -- peuvent rendre pareillement malades ceux qui les ont mis à mort -- et mangés --, aussi le devin ordonne-t-il souvent de leur élever un autel spécial et de leur rendre un culte. De même certains oiseaux de nuit (pi Zgawak, en mofu) peuvent frapper de stérilité la femme qui a écrasé par inadvertance un de leurs oeufs -- ils font leur nid dans le sol -- et ils doivent être conciliés par des autels et des sacrifices. Les esprits de jwoxux font enfin partie de ces esprits à apaiser : les parents de jumeaux sont tenus de rendre un culte à l'esprit de leurs propres enfants, doués d'une force particulière, ambivalente, malëfique ou bẻnéfique. Après une grande fête de sortie des jumeaux -- jusque 1à enfermés -- un autel est élevê sous la direction d'autres parents de jumeaux plus âgês, et des sacrifices y sont offerts chaque année.

Par l'expression génies de possession, on peut rassembler trois types de génies assez différents : les premiers inspirent les devinsguérisseurs mbiala et sont appelés "enfants des devins" ; ils sont seuls présentés comme des génies des montagnes et leur possession est ressentie comme calme et heureuse. Le culte qui leur est rendu est un culte d'alliance, entretenu par des sacrifices réguliers; offerts par les seuls devins-guérisseurs mbidla, ils ne concernent qu'une toute petite fraction de la population. Les seconds gênies de possession, ou facazao, sont censés se rencontrer en plaine. Cette croyance ancienne, semble-t-il, s'est brusquement développée, il y a quarante ans environ, sous l'influence de croyances très proches empruntées aux Giziga, population de plaine voisine; elle est très répandue aujourd'hui : presque la moitié des Mofu, hommes et femmes, entretient un autel à ces génies. Divers degrés peuvent être constatés parmi les fidèles. Les simples fidèles, agressês par une maladie, rendent un culte destiné à les libérer de l'emprise du génie ; celui-ci est dirigé par une initiatrice dont la présence est nécessaire à tous les sacrifices. 
L'organisation de danses pour leurs génies permet à quelques-uns de passer au stade de la possession maîtrisée, de voir leurs esprits et de se libérer de leur initiatrice. Parmi les anciens danseurs, on trouve quelques possêdés-devins, toujours femmes, qui non seulement maîtrisent mais utilisent leur possession; elles peuvent dialoguer avec leurs gênies lors des séances de divination, mais ne sont jamais guérisseurs. Cette possession par les facalao est mal supportée, même par les femmes-devins.

Les génies chitene constituent la troisième espèce de gênies de possession ; génies de plaine à nouveau, dont la croyance, encore peu répandue, a été empruntée aux Peuls voisins. Elle est très proche de la croyance aux facalao et encore plus mal acceptêe. Là aussi il existe une distinction entre les simples fidèles agressés par les génies et les initiateurs les guidant dans leurs sacrifices; il s'agit d'hommes cette fois, à qui la possession ne permet jamais de devenir devins. Les divinités de la montagne ou mbolom ont un pouvoir plus important que celui des gënies de possession car il s'étend à un territoire déterminé -- une portion de montagne --, ainsi qu'au groupe humain établi sur ce territoire. Ce sont des divinités chtoniennes, gênies des lieux existant antêrieurement aux hommes, liées au premier clan venu s'installer sur leur domaine et ayant adopté les autres clans venus par la suite. En elles viennent se fondre les esprits des ancêtres les plus éloignés des vivants, non pas tous les ancêtres mais seulement ceux appartenant au clan défricheur. Elles sont donc composites. Les humains peuvent parfois nouer avec elles des relations directes, entretenues par des sacrifices individuels, mais il s'agit de cultes non-transmissibles. Par contre, chacune a son desservant attitré dont la fonction est hérêditaire et grâce à qui elles peuvent se révêler bénéfiques pour tout un groupe.

La croyance en un Dieu suprême, moteur et fondement de 1'univers, vient coiffer la croyance aux divinités de la montagne. Ce "chef d'en haut", bi erloom, s'intéresse directement aux hommes et on pense qu'il intervient constamment dans leur destinée. Cette croyance très vivante, bien explicitée et antérieure à l'arrivée des Peuls dans la région, ne se traduit pourtant qu'une fois par an lors d'un seul sacrifice. On ne rencontre pas d'autels élevés à ce "chef d'en haut", à la différence, par exemple, de ce qui se passe chez les Dogon (9). 
II. Le sacrifice aux ancêtres.

Pour aborder la description du sacrifice mofu, j'ai choisi le sacrifice aux ancêtres. Il est en effet le plus répandu, le plus lié à la vie de la société. Il montre en même temps le caractère particulièrement concret et vivant de cette croyance aux ancêtres qui, pour être fort répandue en Afrique noire, n'en est pas moins rarement explicitẻe.

\section{A. Les précombules au sacrifice.}

Tout sacrifice a lieu après une consultation divinatoire. Sauf dans les cas de sacrifices familiaux, liés au cycle du mil, où le sacrifiant effectue lui-même sa divination par pailles ou pattes de poulet (10), on a généralement recours à un devin-spécialiste. Dans le cas de sacrifices familiaux, le choix est possible entre un devin inspiré par un génie mbidza -- homme ou femme, il représente le devin traditionnel le plus estimé -- et un devin inspiré par un génie facalao, une femme-devin alors. Le recours au devin est dêterminé par un signe -- une maladie, un dérèglement de 1'ordre naturel (par exemple, un taureau qui se sauve) -- interprété ensuite par le spécialiste comme nécessitant un sacrifice : en ce cas, c'est à la suite de son intervention qu'est offert le sacrifice.

Dans le cas de sacrifices à portée collective -- adressés à une divinité de la montagne, par exemple --, seul un devin mbiala peut être consulté: Le responsable du sacrifice veut savoir s'il n'y a pas d'obstacle à cette célébration : la plupart du temps, le devin décrète alors une série de sacrifices familiaux que le responsable devra offrir à tel ou tel ancêtre; sacrifices secondaires mais qui, tant qu'ils ne ne sont pas offerts, bloquent le grand sacrifice. En ce cas, la consultation divinatoire ne porte pas sur la décision elle-même de ce sacrifice; elle permet seulement de lever les derniers obstacles et de

(9) D. LIFSZYC et D. PAULME, Les fêtes des semailles en 1935 chez les Dogon de Sanga, J. Soc. Africanistes, VI, 1 (1936), pp. 95-110; cf. p. 95 .

(10) J.-F. VINCENT, Divination et possession..., op. cit. pp. 79-89. 
fixer une date.

Lorsque le sacrifice est décidé, les divers participants sont tenus d'observer une abstinence sexuelle (11), particulièrement rigoureuse pour les acteurs principaux du sacrifice, pour lesquels elle s'étend à plusieurs jours. Cette interdiction montre que les relations sexuelles entraînent par elles-mêmes une souillure, madama, qui est insupportable aux puissances supra-naturelles. Notons d'ailleurs que les ancêtres ne sont pas les seuls à manifester cette répugnance ; les gênies de possession et les divinités de la montagne sont censés ne pas accepter un sacrifice -- ne pas "manger"la nourriture offerte -auquel participerait un seul homme ayant eu des relations avec une femme. Le domaine de la sexualité et le domaine religieux apparaissent comme deux domaines sêparés, dont la disjonction devient rigoureuse au moment du sacrifice. Ceux qui n'ont pas respecté $I^{\prime}$ 'abstinence sexuelle ne peuvent pas s'approcher des autels des ancêtres; ils ne pénètrent pas dans la salle des greniers, où a lieu le sacrifice. Ils peuvent juste se tenir dans le vestibule d'entrée : leur part de bière des sacrifices est mise dans une calebasse séparée et ils la boivent dehors. Cette répugnance des ancêtres envers les relations sexuelles apparaît encore dans 1'interdiction qui est faite à la fille de la maison, une fois mariêe, de préparer la nourriture des sacrifices offerts par son père. Cette exigence ne concerne d'ailleurs pas seulement les relations sexuelles mais s'étend au domaine de la sexualité en son entier puisque toute femme ayant ses règles, même si elle est la cuisinière en titre des ancêtres, ne peut faire cuire leurs offrandes. Ces diverses interdictions montrent bien que pour les Mofu l'abstinence sexuelle n'est pas un moyen pour le sacrificateur de rejoindre le sacrê, comme le pensent H. Hubert et M. Mauss (12), elle souligne seulement la différence de nature entre sexualité et sacré.

Afin de mieux disjoindre sexualité et sacrifice, il arrive souvent,

(11) Exigence souvant signalêe ailleurs. Chez les Zaghawa du Tchad, par exemple, elle est requise avant un sacrifice de demande de pluie (M.-J. TUBIANA, Survivances préislamiques en pays Zaghawa (Paris, Inst. d'ethnologie, 1964), 229 pp. (Trav. et Mém. Inst. ethnol., LVII) ; cf. pp. 133,138 ).

(12) H. HUBERT et M. MAUSS, Essai sur le sacrifice, op. cit. p. 49. 184 
lors de sacrifices à portée collective chez le chef de montagne, que le devin décrète une purification préventive, exécutêe par le lignage chargé de purifier la maison du chef, pour le cas oì une souillure, madoma, y aurait été apportêe à l'insu de tous.

Cette purification doit-elle être considérée comme un sacrifice ? Non, bien qu'elle se fasse avec un animal tué spécialement pour la circonstance -- poulet, daman, lézard, plus rarement mouton --, car il n'y a jamais offrande de cet animal. La purification est conçue par les Mofu comme un acte quasi-mécanique :

"On dit que madama est dans la case exactement comme les toiles d'araignée que 1 'on arrache de temps en temps avec des tiges de mil. Pour madoma, c'est pareil, on l'enlève, mais avec la viande d'un animal ; cette viande nettoie madama." (13).

Ainsi les Mofu rejoignent-ils... R. Girard, pour qui la purification religieuse est semblable à un aspirateur -- comparaison inattendue mais soulignant bien ce caractère automatique -- que la ménagère passe dans les moindres recoins de son appartement (14).

On peut distinguer plusieurs degrés et plusieurs espèces dans la souillure, ou madoma. Règles et relations sexuelles constituent, on l'a vu, deux madoma courants. Certaines relations sexuelles illicites entraînent une souillure particulièrement grave, qui peut être durablement incompatible avec le sacrifice : c'est ainsi que j'ai observé un cas où, à la suite d'un adultère ancien avec la fille de son oncle maternel, un chef de famille ne pouvait plus offrir lui-même les sacrifices à ses ancêtres mais devait se faire remplacer par un jeune enfant. Elles peuvent aussi être un obstacle à l'efficacité d'un sacrifice offert pour une collectivité : en 1973, l'absence de résultat d'un sacrifice de pluie à Wazan fut interprétée par le devin comme la consêquence d'adultères non identifiēs, dont la montagne dut se purifier collectivement afin que les ancêtres du chef et la divinité de la montagne laissent à nouveau passer les pluies.

Le domaine de la mort ne peut lui non plus interférer avec le sacrifice. Aussi 1 'enterrement de notables -- devant être pleurés dignement -- retarde-t-il la cêlébration d'un grand sacrifice; s'il s'agit de gens peu importants ou d'enfants le sacrifice a lieu mais

(13) Mazogwoy (40 ans ?), Wazan, 3/1/1970.

(14) R. GIRARD, La violence et le sacré (Paris, Grasset, 1972), p. 398. 
l'enterrement doit être fait clandestinement : il est interdit de battre les grands tambours de deuil habituels. Lors de sacrifices familiaux, celui qui s'est rendu à un enterrement doit attendre quatre jours complets avant de pouvoir boire la bière des sacrifices à l'intérieur de la maison; à la rigueur, il peut en boire à l'extérieur.

Pourquoi cette. séparation entre. la mort et le sacrifice, se traduisant par une attribution aux ancêtres de 1 'horreur des funérailles ? I1 m'a été difficile d'obtenir des précisions 1à-dessus. Les rares justifications recueillies mettent en avant non pas la souillure mais la contagion de la mort, perceptible par ailleurs à travers les rituels d'enterrement et de levée de deuil, qui comportent des lavements et utilisent divers symboles de rupture.

"C'est celui qui est en vie qui offre le sacrifice; il ne doit donc pas avoir participé à un enterrement." (15).

Et puis, pensent les Mofu, il ne faut pas mélanger la joie du sacrifice et la tristesse de la mort.

"On appelle les ancêtres pour leur offrir de la nourriture; il ne faut pas qu'en venant ils trouvent des pleurs de mort !" (16).

Le même informateur poursuit :

"Les ancêtres se fâcheront parce qu'eux sont déjà installés alors que ce nouveau mort n'est pas encore reçu parmi eux. Ils ne veulent pas qu'il soit confondu avec eux."

Les ancêtres sont passés par ce pénible état intermédiaire, mal défini, qu'est la mort. I1 ne faut donc pas le leur rappeler.

B. Nature de l'offrande : sacrifices non-sanglants et sacrifices sangiants.

Pour qu'il y ait sacrifice, faut-il nécessairement qu'il y ait effusion de sang et victime animale ? Les Mofu semblent considérer que non, puisqu'ils possèdent divers sacrifices non-sanglants, à base végétale; chez eux, tout sacrifice n'est donc pas forcément violence.

(15) Sariya (70 ans ?), Wazan, 12/11/1971.

(16) Bletey (65 ans ?), Wazan, 9/4/1970. 
Parmi ceux-ci figurent essentiellement les offrandes de bière de mil, effectuées le plus souvent sous forme de libations (mepesey, 1itt. "verser"). Elles ont lieu avant toute consommation de bière, même de bière non-sacrée. Aussi sont-elles frêquentes puisque la commercialisation de la bière est de plus en plus répandue, mais c'est une bien faible quantité de bière qui est versée à terre par le chef de maison: quelques gouttes seulement, accompagnées d'une courte prière prononcée à haute voix. L'usage mofu rejoint donc celui du monde grec, récenment mis en évidence par É. Benveniste (17); là aussi la libation consistait à offrir à la divinité une toute petite quantité d'un liquide dont la majeure partie était consommée par les hommes. En un seul cas, un sacrifice régulier aux ancêtres est uniquement à base de bière ; il porte d'ailleurs le nom de "bière d'ouverture de grenier", zom ma mi $m a d o$, et est offert chaque année au père défunt par le chef de maison avant de commencer son "mil des hommes". Il arrive parfois que la bière de mil soit remplacée par. une bouillie claire de farine de mil et d'eau, offerte à l'esprit-gardien de la porte sur conseil du devin, cette bouillie représentant un substitut de bière, une formule de sacrifice express.

Un autre cas de sacrifice non-sanglant est constitué par une offrande de boule de mil, accompagnée... d'un os. I1 s'agit là d'un sacrifice très fréquent, décrétê par le devin soit avant un sacrifice important pour apaiser un esprit irrité, soit pour faire cesser une petite maladie. I1 s'agit, comme pour la bouillie de farine de mil, d'un sacrifice express et aussi d'un sacrifice substitut. L'os - - de chèvre ou de taureau -- a été mis de côté lors de sacrifices précédents et ne peut servir qu'une fois.

L'emploi d'un os montre bien que pour les Mofu le vrai sacrifice aux ancêtres est le sacrifice sanglant (sacrifice d'un animal, le sacrifice humain n'ayant jamais été pratiqué, ni pour les ancêtres, ni pour aucune puissance supra-naturelle). L'animal sacrifié peut être un poulet, une chèvre, un mouton ou un taureau, la grosseur de la victime traduisant, selon les Mofu, I'importance de l'ancêtre. Une spécification est discernable : le poulet est offert à l'esprit gardien de

(17) E. BenVeniste, Le vocabulaire..., op. ait. II, p. 220. 
la porte et à l'esprit des champs ; la chèvre au père défunt ; le mouton -- précédé d'un poulet -- est destiné aux ancêtres collectifs de lignage. Dans tous ces cas, I'animal offert peut être minuscule, un poussin, une "chèvre" d'une semaine. Comme l'expliquait un informateur :

"Les ancêtres ne mangent pas vraiment, donc ils ne diront rien. De plus, eux-mêmes savent bien que c'est difficile de trouver une grosse bête !" (18).

Par contre, dans le cas du taureau -- offert à l'ensemble des ancêtres individuels dont chacun reçoit une part -- il ne peut s'agir d'un petit animal. Du moment qu'on s'engage dans ce sacrifice redoutable mais facultatif, il faut le faire à fond.

C. Les agents du sacrifice.

H. Hubert et M. Mauss distinguent entre "sacrifiant", celui pour qui on offre le sacrifice, et sacrificateur, celui qui exécute ou dirige le sacrifice, et ils font remarquer que les sacrifices où le sacrifiant se retrouve seul en face de la victime sont finalement fort rares (19). Chez les Mofu, dans le sacrifice aux ancêtres cette situation n'apparaît jamais. On rencontre, au contraire, en plus du sacrifiant, une vêritable équipe sacerdotale dont la composition varie suivant les types de sacrifices.

Pour les sacrifices familiaux ordinaires, la présence de trois personnes est nécessaire. Est présent tout d'abord " 1 'homme de sacrifice" ou ndo kuley. Chaque chef de maison a en effet son sacrificateur attitré. Des liens de réciprocité existent entre les deux hommes et lui-même sert de ndo kuley à cet homme. Le ndo kuley est un homme de la même classe d'âge et du même clan que le chef de maison; il est souvent un cousin paternel. Sa présence est obligatoire : on retarde ou remet le sacrifice auquel il ne peut assister. I1 est le substitut du maître de maison, à 1 'enterrement duquel il jouera un rôle très important, en particulier lors des dernières offrandes faites aux

(18) Polkwo (50 ans ?), Wazan, 13/1/1970. 48,52 .

(19) H. HUBERT et M. MAUSS, Essai sur le sacrifice, op. eit. pp. 37, 188 
ancêtres du défunt : c'est lui qui clôturera le culte, marquant ainsi la première étape de la transformation du défunt en ancêtre. Son rôle lors des sacrifices consiste à égorger l'animal, poser les offrandes sur les autels, verser la bière, répartir les parts de viande et aussi prier à haute voix.

Un parent âgé, représentant de la génération du père du sacrifiant, un baba, est également présent à chaque sacrifice. Il est surtout chargé de prier le premier et de la façon la plus détaillée. Il peut, lui, changer à chaque sacrifice.

Enfin, un des fils du chef de maison -- ni l'aîné, ni le cadet -est le servant, l'aide. C'est lui qui dépouille l'animal, fait le vaet-vient entre la cuisine et le lieu du sacrifice, va porter la bière aux gens du dehors, suit les ordres du ndo kuley.

Cette équipe restreinte est renforcée lors du sacrifice familial extraordinaire ou maray, ce qui entraîne certains changements dans le rôle des trois participants de base. Pour le maray en effet, il existe un seul sacrificateur par lignage, le doyen du lignage, qui va de case en case pour mettre à mort les taureaux offerts : ce n'est donc plus, en principe, le ndo kuley qui égorge. Mais parfois le sacrificateur de lignage est si âgé qu'il ne fait qu'esquisser la mise à mort et c'est le ndo kuzey qui la termine. De plus la présence de deux neveux utérins, les bizidem, "enfants de soeurs", est requise; ce sont eux qui ont rôle de servants. Rôle important car eux seuls peuvent ranger dans un grenier à part la viande du taureau sacrifiê. Ainsi apparaît la portêe de ce sacrifice hors-série qui permet de rassembler toute la famille restreinte, y compris les filles mariêes en d'autres lignages,présentes par un de leurs fils.

Pour les sacrifices collectifs aux ancêtres du lignage, l'équipe sacerdotale se retrouve à nouveau sous sa forme simplifiée mais par contre les sacrifiants sont nombreux : tous les chefs de famille du lignage ou fragment de lignage ayant le même autel, le même Zudara, doivent être en effet présents.

Quelle que soit leur identité, quel que soit le type de sacrifice, le costume des participants est le plus traditionnel possible : pas de chaussures, pas de vêtements importés. L'idéal est de remettre alors la culotte de peau d'autrefois et de se présenter aux ancêtres torse nu, dans la tenue même qui ètait la leur. 


\section{Jeanne-Françoise Vincent}

Tous ces sacrifices, du plus humble au plus solennel, nécessitent un ensemble de plusieurs personnes, parfois un grand nombre. Pourquoi cette abondance de participants ? Une explication m'a été fournie par un vieil informateur :

"Les ancêtres sont 1à à parler entre eux ; c'est pourquoi il faut que nous aussi, les vivants, nous soyons plusieurs à leur parler !" (20). On peut penser aussi qu'elle est liée au caractère non-écrit de la liturgie : puisqu'il y a un ordre à respecter dans le sacrifice, des. gestes à exécuter, des parts à répartir, le tout d'une façon déterminée, il est nécessaire d'être plusieurs avec si possible des responsables. Ainsi est-on sûr que le sacrifice sera accompli dans les formes et donc qu'il sera accepté par les ancêtres. Pour les Mofu, en effet, l'efficacité des pratiques semble liée à la perfection de leur exécution. Ainsi que le faisait déjà remarquer É. Durkheim, on trouve I'idêe, dans beaucoup de religions, que les rites agissent de façon presque mécanique, "ce qui explique l'importance primordiale attachée (...) à la partie matérielle des cérémonies" (21).

D. Le déroulement du sacrifice.

C'est ici que cette notion de liturgie apparaît avec évidence : les Mofu ont élaboré un schéma du sacrifice aux ancêtres qui se retrouve avec quelques variantes à travers tous les sacrifices et que les participants sont soucieux de respecter. On peut y discerner deux grandes séquences, l'une culminant dans la mise à mort, l'autre dans l'offrande.

La première séquence débute par une prière d'exposition assez longue, prononcée par un parent âgé pour les sacrifices individuels, par le responsable du culte de lignage pour les sacrifices collectifs. Cette prière s'adresse à 1 'esprit concerné, lui rappelle pourquoi et comment il y a eu sacrifice et elle est en même temps une demande d'acceptation.

(20) Kadegal, Wazan, 26/8/1973.

(21) É. DURKHEIM, De la définition du phénomène religieux, L'année sociologique (1899), pp. 1-18; cf. p. 15 . 
C'est la prière la plus dêveloppêe de toutes celles qui seront prononcées par la suite (22). Voici, à titre d'exemples, quelques prières d'exposition ; la première fut prononcée chez Matubay, lors d'un sacrifice au père défunt $(19 / 12 / 1969)$ :

"Le devin nous l'a dit : Matubay a gaspillé la viande et il ne t'a pas encore offert son sacrifice de "fin de grenier". I1 te paie avec la chèvre, le mil, les os. Qu'il reste en bonne santé avec sa famille. Voici la chèvre que tu avais demandée. Fais qu'elle s'ébroue bien !"

Voici une autre prière prononcée chez Užen, lors d'un sacrifice au père également (Gem̌̌ek, 12/1/1971) :

"C'est moi, Užen, qui te parle. Quand j'êtais chez le devin tu as dit : 'I1 faut que mon fils m'offre une chèvre !' La voici, c'est ton petitfils, Taźambat, qui me 1 'a donnêe. A chaque fois je t'ai donné ta chèvre, mais toi, tout ce que je t'ai demandë en $1^{\prime}$ égorgeant, tu ne me 1 'as pas donné ! Mes fils sont mécontents! Ils ne veulent plus cultiver; ils ne veulent plus donner 1 'herbe aux bêtes car ils ne parviennent pas à se marier. Si après 1 'offrande de cette chèvre, mes enfants ne se marient pas, je ne te donnerai plus rien ! Père, vois ! On t'a fait de la bière, c'est pour te demander la femme, la femme, toujours la femme et les enfants. Moi, je suis déjà vieux ! Je ne peux plus travailler !"

Voici encore une autre prière, prononcée chez Waka (Durum, 4/4/1968), lors d'un sacrifice à 1 'esprit gardien de la porte :

"Bientôt, ça va être le moment de semer le mil. Il faut que Waka reste en bonne santé et qu'il trouve beaucoup de mil, mon esprit gardien. Fais qu'il épouse une autre femme et que s'il part en voyage, il fasse un bon voyage! Fais aussi que les maladies ne 1'attaquent pas."

Après cette prière a lieu une courte divination sans paroles dans les sacrifices de poulet, de chèvre et de mouton. Pour le poulet, c'est sa mort même qui est acte divinatoire : on lui tranche la gorge, puis on examine les pattes que la mort a figées dans une position déterminée et signifiante. Pour les sacrifices individuels, la divination porte sur l'année à venir. Ainsi, lors du mêne sacrifice offert à 1'esprit gardien de la porte, le sacrificateur commenta :

"C'est bien ! Il n'y aura pas de maladies chez toi; tous les gens de ta maison et toi-même seront en bonne santé."

Pour les sacrifices aux ancêtres de lignage, la divination par le poulet

(22) Cf. infra, p. 197. 
montre si on peut ou non égorger le mouton qui sera sacrifié ensuite : elle n'est donc qu'une étape.

Si c'est une chèvre ou un mouton qui sont offerts, il faut les arroser d'eau et attendre qu'ils s'ébrouent. Un peu de cette eau est versé au préalable sur les autels. Cet ébrouement constitue d'abord la deuxième partie de la divination amorcée par l'examen des pattes de poulet qu'il vient renforcer. Et surtout il représente l'acceptation des ancêtres, ainsi que le montre une réflexion notée lors d'un sacrifice de Zudara (chez Polkwo, Wazan, 13/1/1970), où le mouton ne se secouait pas et où les assistants s'interrogeaient sur cette absence de réaction :

"Attendez! Les ancêtres sont en train de se parler entre eux. Ils se demandent s'ils vont accepter le mouton."

Cette réflexion montre en même temps que les ancêtres sont conçus comme rassemblés au moment du sacrifice, tout proches de leurs descendants. L'animal à sacrifier devient alors un trait d'union entre le monde des vivants et celui des ancêtres ; les deux sont grâce à lui en communication. Pour les Mofu comme pour les Ndembu observés par V. W. Turner la victime est déjà transformée en envoyé des êtres surnaturels (23). Faut-il en conclure comme lui que c'est avant le sacrifice que les ancêtres -- les "ombres", comme il les appelle -- sont invoqués avec le plus de ferveur ? Oui, si l'on en juge par la longueur de la prière mofu. Toutefois, d'autres étapes du sacrifice constituent, on le verra, des moments aussi importants dans cette mise en relation des vivants et des ancêtres.

Lorsque c'est un taureau qui est offert, il n'y a pas de divination au moment de la mise à mort, ce qui est assez surprenant, étant donné l'importance de ce sacrifice, mais qui s'explique peut-être par les divinations préalables qui ont déjà eu lieu les jours précédents : chaque chef de famille a rempli les instructions du devin et suppose ses ancêtres bien disposés. A moins que le caractère de ce sacrifice, cyclique donc nécessaire, rende inutile le recours à la divination.

(23) V. W. TURNER, Les tambours d'affliction, analyse des rituels chez les Ndembu de Zambie (Paris, Gallimard, 1972), 368 pp. ; cf. p. 308 . 
Chaque famille restreinte possède un matériel liturgique, plats de bois, bols à sauce, calebasses, couteau enfin, sans manche et fait de fer autochtone, qui sert uniquement à mettre à mort les animaux sacrifiés. Il faut que le sang coule (au contraire, dans les sacrifices aux génies de possession, aucune goutte de sang ne doit apparaître et la victime est étouffée en lui enfonçant la tête dans le sable de 1'autel). S'il s'agit d'un poulet, la gorge est tranchée, on l'a vu, et, tandis que l'animal se débat pour mourir, le sang coule partout à terre. La mise à mort d'une chèvre, d'un mouton ou d'un taureau est beaucoup plus solemelle. Elle a lieu en silence -- silence des participants, silence de 1 'animal muselé dont aucun gémissement ne doit se faire entendre car il constituerait un présage de mort. Il existe un mode de mise à mort particulier aux sacrifices aux ancêtres : tandis que la bête est maintenue par des aides -- la bête étant debout s'il s'agit d'une chèvre ou d'un mouton, couchée sur le côté et pattes entravées s'il s'agit d'un taureau -- le sacrificateur perce la veine jugulaire avec la pointe du couteau sacrificiel. L'animal est véritablement poignardé, alors que dans la vie courante, sur les marchés par exemple, on lui tranche la gorge. Il semble qu'il y ait là volonté de souligner par ce détail l'acte religieux. Le sang de la victime est recueilli dans un plat de bois ou un bol de terre où il coagule; on le place alors un instant sur les autels. La chèvre -- ou le mouton -est ensuite posée sur les autels, où elle finit de mourir, "pour montrer aux ancêtres qu'ils vont bientôt avoir leur part". De légères onctions de sang sont faites sur les poteries des ancêtres tandis que les participants se mettent du sang sur le front. C'est là la seule utilisation de ce sang frais sur lequel le sacrifice mofu, d'une façon générale, n'insiste guère. On ne trouve pas ici l'idée, courante pourtant en diverses sociétés africaines, chez les Dogon par exemple (24), suivant laquelle le sang d'une victime constitue la partie privilégiée de l'offrande, si bien que faire un sacrifice consiste d'abord à répandre du sang, ce sang qui est bu par la divinité ou l'esprit concerné. Peut-être faut-il voir dans les onctions de sang du sacrifice mofu

(24) D. PAULME, L'organisation sociale des Dogon (Paris, 1940); cf. p. 428 . 
1a volontê de relier les agents du sacrifice aux ancêtres par l'intermédiaire des autels ? Une partie du sang coagulé est mélangée à de la farine de mil et mangée en silence par les participants les plus âgés, ce qui pourrait être considéré comme un rite de communion mais demande à être vêrifié.

L'animal tué est ensuite préparê. Un poulet est plumé, vidé et passé à la flamme. Chèvre, mouton et taureau sont dépouillés de leur peau. Comme pour la mise à mort, cette opération qui pourrait être banale est placée dans un contexte religieux par des détails précis : il existe une façon religieuse d'enlever la peau des animaux offerts ; au mouton et à la chèvre, on laisse une bande de peau qui va du museau, oreilles comprises, à la pointe de la queue et est enlevée en dernier. De plus, on n'ouvre pas les côtes et on détache les pattes de devant en même temps que la peau. Une bande de peau est également laissée au taureau et enlevée la dernière; cette fois il s'agit de la peau du ventre entre les pattes, portant les testicules. Par ailleurs, au fur et à mesure qu'on dépouille l'animal, on le saupoudre de farine de mil, ce qui ne se fait pas en dehors des sacrifices.

Une attention particulière est portée au contenu de l'estomac, au rumen, qui sert aussitôt à asperger les autels, littéralement encroûtés par les traces des précédents sacrifices. Pourquoi cette importance du rumen ? Elle vient selon les Mofu de sa permanence : il ne pourrit pas et peut subsister des mois durant. Il constitue donc une trace matérielle du sacrifice destinée, selon eux (25), à la fois aux ancêtres eux-mêmes -- qui ne rêclameront pas trop vite un nouveau sacrifice -- et aux sorciers -- qui hêsiteront à pénétrer dans une maison dont les ancêtres sont satisfaits, donc pleins de vigilance.

La peau, complètement enlevẻe, est placée un moment sur l'autel pour que I'ancêtre concernê puisse suivre la progression du sacrifice.

C'est alors qu'intervient le partage de la victime. I1 s'agit lã d'une opération complexe et particulièrement signifiante qui pourrait donner lieu à de longs développements. En résumé, l'ancêtre que l'on veut honorer doit recevoir des fragments prélevés seulement sur certaines parties de l'animal : il existe un code valable pour chaque

(25) Explications d'Erlamaka (Durum, 15/8/1973). 
bête en qui on distingue des parties valorisêes, pouvant être offertes aux esprits, et des parties profanes, destinees seulement aux vivants. Sur un poulet, par exemple, on prendra la cuisse, 1'aile, la tête ; sur une chèvre offerte au père défunt, on détachera des fragments de patte avant gauche, de foie, d'intestins, de côtes avec vertèbres ; sur un mouton offert au Zudara, on prélèvera la tête, un peu de côtes, de foie et de poumon. Ces morceaux sêlectionnés ne peuvent être offerts tels quels : ils subissent nécessairement une cuisson qui a lieu sous deux formes ; une partie de la viande à offrir est grillée sur le champ par les hommes; 1'autre est remise aux femmes pour être préparée en sauce.

Par cette obligation de cuisson de la victime le sacrifice mofu apparaît comme un repas sacrificiel que l'on retrouve en diverses sociêtés africaines, les Zaghawa par exemple (26). On pourrait même diviser les ethnies africaines en celles qui offrent crue la viande des sacrifices et celles qui l'offrent cuite. Chez les Mofu, cet aspect de repas sacrificiel est particulièrement apparent puisque la viande cuite ne peut être offerte seule. Il faut l'accompagner de boule de farine de mil et de libations de bière de mil. Cet appel à une préparation culinaire fait d'un sacrifice sanglant aux ancêtres un acte long, se déroulant le plus souvent sur une journée complète. Pourquoi la viande cuite doit-elle être associée à de la boule ? Cette obligation est particulière aux ancêtres; aux génies de possession, par exemple, on offre de la viande cuite sans rien d'autre. Peut-être s'exp1ique-t-elle par 1e fait que pour les Mofu l'existence post-mortem ne fait que prolonger l'existence terrestre : les ancêtres ont donc gardé les habitudes alimentaires des vivants.

La nécessité d'une préparation culinaire ëlaborēe et complexe oblige les Mofu à faire appel aux femmes qui tiennent ainsi une part importante dans les sacrifices masculins. Les offrandes -- viande en sauce, boule de mil, bière de mil -- sortent en grande partie de leurs mains, alors qu'en diverses régions d'Afrique -- chez les Zaghawa, par exemple, pour poursuivre la même comparaison -- les femmes n'ont même pas cette possibilité de participation indirecte. Toutefois ce recours

(26́) M.-J. TUBIANA, Survivances préistomiques..., op. cit. pp. 139, 156, etc. 
aux femmes ne va pas jusqu'à leur permettre de prendre la parole lors des prières aux ancêtres de leur mari ou de leur père. De plus, bien que nécessaire, la participation féminine est surveillée de près : la préparation culinaire doit respecter diverses règles très strictes. Elle nécessite d'abord un matériel spécial, et dans les familles de notables on lui réserve même une "cuisine des sacrifices" à part qui ne sert donc que quelques jours dans l'année. Les enfants sont écartés ; si l'un d'eux touchait l'un des ustensiles de cuisine ou, pire, goûtait à la nourriture du sacrifice, celui-ci, à coup sûr, serait refusé par l'ancêtre. La façon de cuisiner les offrandes est, elle aussi, particulière; c'est ainsi qu'elle ne doit pas comporter de sel. Quant à la préparatrice, elle ne peut varier. Elle est soit la mère du chef de maison, soit une de ses filles encore célibataire; à défaut, ce peut être sa femme, et s'il est polygame, sa première femme. Lorsqu'il faut la changer, on organise un sacrifice spécial pour en aviser officiellement les ancêtres, qui sinon risqueraient de se fâcher. La préparation des offrandes se fait avec une véritable hantise de la colère des ancêtres, hantise qui se rêvèle lors des consultations divinatoires.

La présentation des offrandes préparées a lieu en plusieurs temps suivant un ordre déterminé. Offrande de la viande grillée par les hommes accompagnée de boule, puis libations facultatives de bière, et, pour clôturer le sacrifice, après un laps de temps parfois long, offrande de viande en sauce et de boule. Grillée ou en sauce, la viande est offerte de la même façon : le servant divise la boule en plusieurs parts, chacune portant collé à elle un morceau de viande. Ces parts -- en nombre pair ou impair suivant l'identité de l'ancêtre destinataire du sacrifice -- sont déposées sur les autels. L'ancêtre concerné reçoit double part, mais chaque ancêtre a la sienne ou, si le sacrifice est peu important, 1 'ensemble des ancêtres reçoit une part collective. En effet, expliquent les Mofu, les ancêtres ne doivent pas être frustrês, ce qui pourrait faire naître en eux un esprit de vengeance. Et puis, on espère aussi que cette petite offrande les satisfera et les empêchera de réclamer trop vite un sacrifice spécial complet. La composition de cette part est codifiée : à chaque esprit d'ancêtre correspondent des morceaux précis variant suivant 1 'animal offert.

Lorsque de la bière a été faite, elle est offerte sous forme de libations; les autels sont arrosés, avec largesse ou parcimonie, 
suivant 1a quantité dont on dispose, et quelques gouttes sont versêes à terre pour les ancêtres lointains.

Cette présentation des offrandes est scandée par des prières prononcées en premier par le sacrificateur, puis par le doyen et par chacun des participants, sacrifiant compris, au gré de son inspiration. L'attitude normale pour parler aux ancêtres -- et pour sacrifier -est la position accroupie ou assise. Il est frappant de constater le contraste entre la liberté laissée à chacun pour ces paroles alors que les gestes et leur succession doivent obéir à un code. Il n'existe pas de véritables formules liturgiques et chacun parle comme il 1'entend en s'exprimant au nom de celui chez qui a lieu le sacrifice.

Les prières sont faites à quatre moments différents du sacrifice et regroupées ici pour plus de commodité. Elles ouvrent, on 1'a vu, le sacrifice (27). Elles accompagnent ensuite la dêpose des offrandes sur les autels. Si celle-ci se fait par étapes, chacune seıa marquêe par des prières qui, cette fois, seront plus nettement des prières de demande, accompagnées d'injonctions à manger la nourriture offerte. Ainsi lors du sacrifice au père dêfunt de Matubay (Wazan, 19/12/1969) :

"Tiens ta boule, avec la viande que tu avais demandée. Mange bien, père! Il faut que Matubay trouve de l'argent avec son coton! Qu'il épouse encore une fernme! Toi, son père, tu avais six femmes. Pourquoi n'en a-t-il pas autant ? Fais aussi qu'il achète des chèvres pour mettre dans son étable ! Tiens, prends la viande qu'il t'a payée !"

Lorsqu'après un certain laps de temps les offrandes sont enlevées des autels, elles sont accompagnées de courtes prières, telle celle prononcêe au même sacrifice chez Matubay :

"Je reprends la boule et la viande. Quand Matubay vendra son coton, qu'il ait beaucoup d'argent !"

Enfin le versement de la bière est l'occasion de nouvelles prières et demandes, entrecoupées d'encouragements à boire :

"Prends la bière et bois bien ! On te donne la viande, la bière, tout ! On t'a tout payé ! Maintenant, il ne faut plus que la maladie revienne. Le mil qui avait êtê perdu, la viande, la bière, on te paie pour tout ! Que tous les habitants de la case de Matubay restent en bonne santé ! Bientôt Matubay battra son mil, fais que son mil augmente ! Qu'il épouse encore une femme et nous aussi ! Bois bien! Toi le père, et vous les autres, buvez !"

(27) Cf. supra p. 191. 
Ces divers exemples font ressortir le caractère concret des prières, qui expriment la conviction de la présence des ancêtres au sacrifice et celle de leur puissance s'étendant à tous lẹs domaines de la vie. On est frappé aussi par leur aspect contractuel, cet aspect que signalait déjà É. Durkheim (28) : l'esprit reçoit sa part, donc on peut avoir prise sur lui. Aucune notion d'amour de l'ancêtre ne s'y exprime; si on lui offre un sacrifice, c'est pour obtenir de lui un résultat précis, mais c'est tout. On ne sent pas non plus de respect, de révérence envers cet ancêtre, qui est traitê, pour reprendre 1 'expression de É. Durkheim, "sur le pied de la plus parfaite égalité" (29).

Parallèlement au repas des ancêtres a lieu celui des vivants. Ceux-ci doivent consommer à la fois la nourriture sacrëe, celle qui a été placée sur les autels, et la nourriture profane, les parties de la victime qui ne conviennent pas à I'offrande; en effet, la totalité de l'animal doit être mangée le jour du sacrifice.

La consommation de la nourriture profane a lieu la première. Pendant que les parts de chaque ancêtre sont encore disposées sur les autels, le sacrificateur distribue aux présents le restant de la viande cuite mais non offerte. Parmi eux, peuvent se trouver des.gens n'appartenant pas au clan. Le sacrificateur mange le premier et tous l'imitent. Ainsi, les vivants mangent au moment où les ancêtres sont censés consommer en esprit leur part. Cette coincidence dans le temps, ce rapprochement dans l'espace, cette communication entre vivants et ancêtres signifient-ils communion? Je peux le supposer mais dois faire remarquer que les Mofu ne l'ont jamais mise en évidence. Au contraire, les Hausa, lorsqu'ils rendent un culte aux divinitês anne et consomment les animaux qui leur sont sacrifiês auraient le sentiment d'une communion alimentaire avec elles (30). Les Mofu font peut-être partie de ce grand nombre de peuples chez qui, ainsi que le remarque E. E. EvansPritchard, "le sacrifice n'a absolument pas le sens d'une communion" (31) ? A moins que ce ne soient les ethnologues qui, influencés par

(28) E. DURKHEIM, De la définition..., op. cit. p. 8.

(29) É. DURKHEIM, Zoc. cit.

(30) J. MONFOUGA-NICOLAS, Ambivalence et culte de possession (Paris, Anthropos, 1972), p. 45.

(31) E. E. EVANS-PRITCHARD, La religion des primitifs (Paris, Payot, 1965), p. 64. 
les penseurs du dẻbut du siècle -- H. Hubert et M. Mauss, pour ne pas les nommer, ou encore R. Smith (32) -- ne soient tentés de solliciter les faits pour y trouver cette communion avec les esprits supra-naturels qui serait liée nécessairement au sacrifice?

Une fois la nourriture profane consommée, restent les parts des ancêtres. Elles ne sont pas abandonnées sur place mais reprises, on l'a vu à propos des prières. Le sacrificateur prélève un minuscule fragment qu'il jette à terre. Pour qui ? Deux explications m'ont êté données : ce fragment serait pour l'ancêtre lui-même (33) dont il constituerait la véritable et bien modeste part ; d'autres informateurs le croient destiné aux esprits d'ancêtres morts sans descendance, attirés par le sacrifice et n'ayant pas d'autels (34). Mis à part ce fragment, la part de viande et de boule va à un membre déterminé de la famille vivante -- celle de la mère du père, par exemple, revient à la fille aînée non mariée du chef de maison -- qui la consomme sur le champ. Cette fois, un étranger au clan ne peut recevoir de cette nourriture sacrée. Là aussi, on pourrait parler de communion avec les ancêtres mais en l'état actuel de mes recherches, mieux vaut m'abstenir.

Ce sont les vivants, on le voit, qui consomment la quasi-totalité des offrandes. Pourtant les Mofu, sans tenir compte du volume dérisoire de la part des ancêtres, considèrent que par le sacrifice ceux-ci ont mangé et sont satisfaits de leurs descendants.

Bien des aspects du sacrifice mofu n'ont pu être traitês dans cette étude, le chômage, ce "culte négatif", comme disait É. Durkheim (35) ou le symbolisme par exemple. Évoquons pour terminer un dernier aspect essentiel : le facteur de cohésion que représente le sacrifice à tous les niveaux de la socięté.

Dans la famille restreinte, les divers liens -- entre mari et femme, père et fils, père et fille -- sont resserrés par la nécessité d'oeuvrer ensemble à la réussite du sacrifice; à l'intêrieur de la

(32) R. SMITH, auteur de l'article "Sacrifice" dans l'Encyclopedia Britannica des années 1890.

(33) Polkwo (Wazan, 13/1/1970).

(34) Matubay (Wazan, 19/12/1969).

(35) E. DURKHEIM, Les formes élémentaires de la vie religieuse ${ }^{4}$ (Paris, PUF, 1960), p. 438. 
parenté il en va de même : paternels, maternels, alliês reçoivent une part de nourriture des sacrifices solennels; à l'échelon de la "montagne", le sacrifice crêe également 1'unité : c'est en cêlébrant leurs sacrifices -- et tout particulièrement le sacrifice du taureau -- ensemble au signal de leur chef, que les "chefs de maison" prennent conscience du groupe social qu'ils constituent; enfin, à travers 1 'ensemble mofu, c'est la forme particulière à leurs sacrifices qui donne aux Mofu le sentiment de former une ethnie distincte. Aussi, en parlant des montagnards Gudur, leurs voisins du sud, ils déclarent : "Là-bas, ils n'ont plus les mêmes sacrifices que nous ; ce ne sont plus nos frères !".

\section{I S C U S I O N}

L. de Heusch : Vous apportez des éléments nouveaux passionnants et tout particulièrement l'idée que le sacrifice obéit à un code culinaire. On retrouve le même souci dans une tribu bantoue d'Afrique australe, les Thonga. Il me semble que dans les différentes phases de la consommation rituelle une série d'éléments prennent leur pleine valeur par leur position dans toute la séquence. Vous avez remarqué vous-même que la consonmation du sang mêlé à la farine se fait en silence avant la consommation des éléments cuits, qui s'accompagne de parole. Tout se passe donc comme si il y avait une volonté de non communication lorsqu'on absorbe le sang, la communication étant liée à la cuisine. Entre ces deux épisodes, se situe un élément assez mystérieux, que je retrouve aussi chez les Thonga, c'est l'utilisation du rumen. Les Thonga opposent une viande bouillie par les hommes à l'intérieur du village et une viande rôtie à l'extérieur par tous ceux qui ne font pas partie du lignage et en particulier les neveux utérins qui sont ici les grands sacrificateurs. J'aimerais savoir quel, est le mode de cuisson rituelle des Mofu après le sacrifice.

J.-F. Vincent : Chez les Mofu, il y a opposition entre la viande rôtie par les hommes à l'intérieur de la salle des greniers, donc auprès des autels, et la viande bouillie par les femmes dans leur cuisine, loin des autels. Sur le mode de préparation de cette viande bouillie, sur la confection de la sauce en particulier, je ne peux apporter qu'une précision, l'absence de se1, car je suis toujours restée du côté des hommes lorsque les femmes la préparaient. En effet, c'est un moment très long et très instructif durant lequel on évoque le souvenir des hauts faits guerriers, on raconte la vie d'autrefois, le statut et la conduite des "gens du chef" par exemple.

L. de Heusch : Ce qui me frappe encore ce sont ces opérations de purification qui font intervenir un animal non domestique, le daman. Peut-on aussi utiliser la chèvre dans ce cas ?

$$
\text { J. }-F \text {. Vincent : Non, la chèvre ne sert pas aux purifications; }
$$


c'est le mouton qui est utilisé. On ouvre l'estomac de ce mouton vivant et on en projette le contenu sur toutes les parties de la maison à purifier. mâles ?

$J$. Rouch : Les animaux offerts aux ancêtres sont-ils tous des

J.-F. Vincent : Il est dans la $\operatorname{logique~du~système~mofu~que~ce~soit~}$ des mâles. Cependant, ayant omis de poser expressément 1a question sur le terrain, j'ai préféré dans mon exposé ne pas donner de précisions à ce sujet.

J. Rouch : Un autre point : la couleur de la robe des victimes, quelle est-elle ?

J.-F. Vincent : Cette couleur est signifiante pour la divinité de la montagne à qui il faut offrir un animal blanc. Elle est signifiante aussi pour les esprits d'hommes tués à la guerre auxquels il faut offrir un animal noir.

A. Adler : Chez les Mundang, il n'y a que le mouton qui doit avoir une couleur déterminée.

J.-F. Vincent : Effectivement, dans ces deux cas, il s'agit de moutons. Je pense à la réflexion qu'une couleur spécifique n'est pas exigée pour les chèvres.

J. Rouch : Chez les Songhay par contre, j'ai vu des sacrifices de bouc où les couleurs étaient bien déterminées ; il y en avait trois : rouge, noir et tacheté. Dans quelle direction sacrifie-t-on ? Sur quel côté l'animal est-il posé ?

J.-F. Vincent : L'animal est généralement couché sur le côté gauche lorsqu'il s'agit d'un taureau ; d'autre part, on le tourne en direction de l'ouest, parce que c'est la direction dans laquelle on chasse l'impureté. On dit : "souillure, souillure, pars vers l'ouest".

J. Rouch : L'animal est-il préparé ? Doit-il être consentant et ne plus se débattre?

J.-F. Vincent : I1 n'y a pas de préparation. On se contente de le maintenir très solidement pour qu'il ne bouge pas, en lui maintenant le museau fermé pour qu'il ne puisse pas émettre un seul gémissement. Par ailleurs, quand il s'agit d'un taureau, on lui ligotte les pattes.

L. de Heusch : Il y a donc volonté d'affirmer I'absence de communication entre $l^{\prime}$ animal et $l^{\prime}$ homme, puisque 1 'un et l'autre doivent rester silencieux.

A. Adler : Vous avez parlé de proscriptions sexuelles avant le sacrifice. N'y a-t-il pas par ailleurs proscription des relations sexuelles avant toute tâche délicate?

J.-F. Vincent : Oui, par exemple, autrefois, avant de fondre le fer, avant d'aller à la guerre ou à la chasse, il fallait s'abstenir de relations sexuelles. On pense d'ailleurs que les jeunes gens qui ne connaissent pas encore les femmes courent moins de risques à la guerre.

A. Adler : Dans votre exposé, vous n'avez pas décrit les autels.

J. $-F$. Vincent : Il s'agit de très petites poteries, sans figuration humaine ni sexuelle, fort peu spectaculaires. Il y a un symbolisme que je n'ai pu aborder ici, c'est celui des nombres qui apparaît 
au moment du sacrifice. Le sexe du premier enfant voue ses parents au pair ou à l'impair. Si le premier-né est un garçon, au moment du sacrifice, certains gestes seront obligatoirement accomplis un nombre impair de fois. Ce sera un nombre pair s'il s'agit d'une fille.

A. AdZer : Ceci est lié à la notion de chance. On a 1a chance du chiffre du sexe de son premier enfant. C'est vrai dans tout le Tchad.

J. $-F$. Vincent : Par ailleurs, chez les Mofu, la main gauche est la main de l'homme ; la gauche est donc valorisée. C'est vers elle que $l^{\prime}$ on se tourne pour offrir les sacrifices aux ancêtres-hommes.

L. de Heusch : Chez les Thonga, la règle sexuelle est formelle : on utilise toujours un animal du sexe opposé à celui du sacrifiant et l'on dit que cette complémentarité sexuelle favorise la fécondité.

A. de Surgy : Y a-t-il des sacrifices au dieu suprême?

J. $-F$. Vincent : Oui, mais ils ont lieu une seule fois par an, au moment de la fête collective appelée "bière de Dieu" ; ils comportent une offrande de bière et de viande sans prëparation culinaire. La viande est offerte à l'écart de l'habitation ; c'est une toute petite part, placée dans un tesson mis sur le bord du chemin. Pour Dieu, chacun tue l'animal que ses moyens lui permettent; cela va du poulet au taureau.

G. Le Moal : $\mathrm{Y}$ a-t-il des sacrifices de chiens ?

J.-F. Vincent : Non, jamais. Cependant les rites destinés à rétab1ir la paix entre deux "montagnes" obligent à tuer deux chiens, qui sont dêpecês, partagés puis enterrés par les "montagnes" réconciliées.

L. de Heusch : Pourriez- vous commenter l'usage de la peau du taureau sacrifié?

$J .-F$. Vincent : Je peux donner quelques précisions non sur l'utilisation de cette petite bande mais sur l'ensemble de la peau. Une moitié revient à la femme du sacrifiant, l'autre à celui qui a élevé le taureau.

M. Cartry : Le port de la peau par la femme a-t-il un rapport avec la fécondité ?

J. $-F$. Vincent : Oui, car cette peau ne peut être portêe que par une femme qui a déjà étê enceinte.

L. de Heusch : Le choix des animaux sacrificiels est-il guidé par la divination ou relève-t-il d'un code automatique ?

$J .-F$. Vincent : Le choix est automatique. C'est un code.

M. Cartry : Y a-t-il des rites sacrificiels sanglants sans consommation de viande ?

J. $-F$. Vincent : Dans les rites de purification, la viande est mangée par le sacrificateur. Je ne vois qu'un cas où la viande n'est pas consommêe, mais où le sang n'est pas versé non plus ; c'est celui des sacrifices de reconnaissance offerts à une divinité de la montagne. En effet, on pense que la divinité peut accorder une femme ou un enfant à celui qui, n'en ayant pas, est allé spécialement dans la montagne 1a supplier. Quand son souhait est exaucé, il doit un animal à la divinité, au mbolom. Il doit lui casser une patte et le lâcher dans la montagne. L'animal finit par mourir mais sa chair n'est pas consommée. Aussi, à la réflexion, je ne sais trop s'il. faut parler de sacrifice, plutôt 
d'offrande.

Luc de Heusch : Les rites de passage sont-ils accompagnés de sacrifices?

J. $-F$. Vincent : C'est le cas pour les funérailles, mais non pour le mariage, sauf si la mariée est née après la constitution d'un autel aux oiseaux de nuit. Le culte se termine avec son mariage et on fait un dernier sacrifice. Au moment de la naissance, il y a des rites mais pas de sacrifices. La participation à un rite funéraire empêche d'être agent actif d'un sacrifice.

Lue de Heusch : Les deux grandes sources d'impureté sont donc la sexualité et la mort.

M. Cartry : On assiste à une disjonction entre le champ religieux et la sexualité. On pourrait dire tout aussi bien une conjonction. Ce n'est pas la sexualité en tant que telle qui est visée, mais l'acte sexuel. On pourrait se demander si ce n'est pas lié à une déperdition d'ênergie incompatible avec la célébration de certains rites.

J.-F. Vincent : Je ne pense pas que cette interprêtation soit valable pour les Mofu car chez eux c'est bien la sexualité toute entière qui est séparée du religieux et non pas 1'acte sexuel; ainsi les femmes qui ont leurs règles ne peuvent préparer la viande sacrificielle.

Luc de Heusch : Il y a des ëquivalents bantous. Pour les Lele, le sperme, le sang menstrue1, les excréments, la saleté relèvent de la même catégorie. La sexualité est aussi polluante chez les Bemba. Elle 1 'est également dans la pensée chrétienne. Pourquoi sinon parleraiton de 1 'Immaculée Conception ?

M. Cartry : Chez les Gourmantché, le sang menstruel n'est pas seulement lié à la souillure, il est d'abord symbole de non-fécondité. Dans de nombreux rites, le trait pertinent pour le sang menstruel est "non-enfant", et dans ce sens il rapproche de la mort.

L. de Heusch : Chez les Mofu, le rumen semble être un élément de rapprochement entre les hommes et les ancêtres, alors que chez les Thonga, il apparaît comme un ëlément de dispersion.

J. $-F$. Vincent : Le rumen $n^{\prime}$ est pas seulement $1 \mathrm{i}$ é aux ancêtres. Il sert aussi à purifier, par exemple on purifie la montagne en projetant du rumen dans sa direction. 